\title{
De que Forma a Cultura do Compartilhamento e Modificação pode Colaborar no Processo de Desenvolvimento de Jogos?
}

\author{
Arison Heltami Rodrigues Uchoa ${ }^{1}$, Emanuel F. Coutinho ${ }^{1}$ \\ ${ }^{1}$ UFC Virtual - Universidade Federal do Ceará (UFC) - CE - Brasil \\ arisonheltami@gmail.com, emanuel@virtual.ufc.br
}

\begin{abstract}
Resumo. O processo de desenvolvimento de um jogo digital passa por diversas etapas, das mais variadas formas, com o objetivo da criação de um produto final mais eficiente. Em geral, essas etapas são: Conceito, Pré-Produção, Produção e Pós-Produção. Crowdsourcing é um paradigma que está gradualmente sendo usado com mais regularidade e com uma ampla gama de propósitos, tais como reunir muitas fontes de conhecimento, cada uma com diferentes niveis de experiência e especialização, para servir a muitos propósitos. O objetivo deste trabalho é avaliar o impacto do uso de estratégias de crowdsourcing no desenvolvimento e manutenção de engajamento em jogos digitais, delimitando como a construção coletiva enriqueceu o produto.
\end{abstract}

\section{Introdução e Motivação}

O advento das redes sociais virtuais e a popularização de diversas plataformas de comercialização e contato entre desenvolvedores e usuários têm promovido, entre outros efeitos, um maior engajamento de participantes externos às equipes de desenvolvimento de jogos em seus processos produtivos. Enquanto alguns tipos de intrusões já são comuns ao processo de desenvolvimento, como por exemplo a busca por feedback de usuários e a avaliação de conceitos e interações mediante testes (os chamados playtests), tornouse cada vez mais possível o engajamento da comunidade como um todo na criação de conteúdos para jogos, dispersando os esforços entre um maior número de participantes para a criação de um produto final de qualidade ou derivados que promovem novas experiências partindo do material original.

Segundo Jeff Howe, "Crowdsourcing é atrair pessoas externas a empresa e envolvê-las em processos amplamente colaborativos e criativos" [Howe 2008]. A perspectiva empresarial de Howe também pode ser adequada para projetos individuais e atividades sem fins comerciais. Assim, o crowdsourcing combina o esforço do público para resolver um problema ou produzir um recurso [Wang et al. 2013].

O crowdsourcing é um paradigma que está gradualmente sendo usado com mais regularidade e com uma ampla gama de propósitos [Carter et al. 2012]. Reunir muitas fontes de conhecimento, cada uma com diferentes níveis de experiência e especialização, através do uso inovador da tecnologia, pode servir a muitos propósitos. O crowdsourcing pode ser considerado como a melhoria da terceirização (outsourcing), já que a terceirização é quando um trabalho é atribuído a um indivíduo definido, enquanto que o crowdsourcing atribui um trabalho a um grupo indefinido e grande de pessoas.

Meios de crowdsourcing (colaboração coletiva) estão entre as principais formas de engajar a comunidade ao redor de uma ideia, popularizando-a dentro de um grupo, criando 
um maior senso de posse e pertencimento que muitas vezes reflete no resultado comercial do produto, seja pela eliminação ou pela redução de diversos obstáculos técnicos e de mão de obra para o cumprimento de requisitos e etapas necessárias na aplicação.

Esta pesquisa tem como objetivo geral realizar uma avaliação do impacto do uso de estratégias de crowdsourcing no desenvolvimento e manutenção de engajamento em jogos digitais, delimitando de que forma a construção coletiva enriqueceu o produto a partir de uma perspectiva qualitativa e comercial. Como objetivos específicos tem-se o mapeamento de estratégias de construção coletiva possíveis para o processo de desenvolvimento de jogos, passando das estratégias mais utilizadas e solidificadas na indústria de desenvolvimento até metodologias com menor amplitude de utilização e o levantamento de projetos que lançaram mão de formas de crowdsourcing, especificando os objetivos para a utilização desses meios dentro da proposta do produto final.

Tal pesquisa possui implicações pertinentes para a indústria de desenvolvimento de jogos, com a construção de uma análise estruturada entre os meios e crowdsourcing, e o processo de desenvolvimento de um jogo. Ela também pode servir de base para a melhor utilização dessas ferramentas em uma perspectiva mercadológica, com uma maior difusão de formas alternativas de desenvolvimento, propiciando a criação de projetos em caráter experimental, gerando uma maior abertura na indústria de jogos, promovendo a troca de conhecimentos entre partes atuantes nesse setor, do desenvolvimento até o consumo.

\section{Trabalhos Relacionados}

[Carter et al. 2012] discutiram as recentes tecnologias emergentes com foco especial no possível uso do conceito de crowdsourcing. Foram discutidos métodos de verificação de qualidade para ajudar no caso do cenário de design de jogos de computadores. Também uma arquitetura para permitir o design de missões em jogos foi apresentada.

[Munezero et al. 2013] exploraram a plataforma de redes sociais Facebook como fonte de dados textuais com anotações de emoção.Contratar especialistas para fornecer dados manualmente rotulados (anotados) para pesquisa em processamento natural de linguagem é demorado, tedioso e caro. Assim, crowdsourcing surgiu como um método útil para obter dados anotados. O trabalho desenvolveu o jogo do Facebook EmotionExpert para coletar dados textuais anotados por humanos e detectar emoções no texto. O jogo fornece um meio para alcançar um grande número de jogadores que realizam anotações do conteúdo emocional dos textos uma atividade social. Descobertas indicam que o jogo é útil para alcançar um grande número de pessoas na produção de anotações confiáveis.

[Shaker et al. 2013] trataram da estética dos jogos de plataforma e o que torna um nível de plataforma envolvente, desafiador ou frustrante. Para isso, utilizou-se mineração de dados de um jogo de crowdsourcing de um clone do clássico jogo de plataformas, o Super Mario Bros. Os dados consistem em 40 níveis com variados parâmetros de design. Coletivamente, esses níveis são reproduzidos 1560 vezes pela Internet, e a experiência percebida é anotada pelos participantes da experiência.Dada a riqueza desses dados de crowdsourcing, à medida que todos os detalhes sobre o comportamento dos jogadores no jogo são registrados, o problema é extrair recursos numéricos significativos no nível apropriado de abstração para a construção de modelos computacionais genéricos de experiência do jogador e, portanto, estética do jogo. Houve um avanço nos fatores que contribuem para a estética do jogo de plataforma, e os resultados foram úteis para a 
personalização da experiência do jogo através da adaptação automática.

Com o rápido desenvolvimento do crowdsourcing em todo o mundo, o crowdsourcing para Engenharia de Software começa a atrair mais atenção de desenvolvedores de software, codificadores e pesquisadores [Hu e Wu 2014]. Muitas plataformas online bem-sucedidas demonstraram a capacidade de crowdsourcing e o potencial para dar suporte a várias atividades de desenvolvimento de software. No TopCoder, para estudar comportamentos competitivos no crowdsourcing de software, aplicou-se teoria dos jogos para modelar desafios de algoritmos, onde codificadores criam desafios. $\mathrm{O}$ artigo fornece uma nova perspectiva de pesquisa para o crowdsourcing de Engenharia de Software.

[Prandi et al. 2016] apresentaram os resultados obtidos em testes de campo usando o jogo Geo-Zombie, que mistura a realidade com zumbis virtuais em um ambiente urbano. Geo-Zombie é projetado para coleta de dados georreferenciados sobre acessibilidade urbana, com o objetivo de envolver um grande número de jogadores na atividade de sinalização durante caminhadas. Para obter munição para reagir a um ataque de zumbis, os jogadores podem sentir / mapear (crowdsensing) barreiras e instalações urbanas, transmitindo esses dados a um centro operacional (crowdsourcing). Os resultados confirmam a viabilidade e adequação da abordagem e estimulam discussões interessantes.

[Rojas et al. 2017] apresentou uma abordagem baseada em gamificação e crowdsourcing para produzir melhores testes de software. O jogo web CODE DEFENDERS permite que equipes de jogadores disputem em um programa, onde os atacantes tentam criar defeitos sutis, e os defensores tentam contrapor escrevendo fortes testes. Experimentos em cenários controlados em crowdsourcing revelam que escrever testes como parte do jogo é mais agradável, e que jogar CODE DEFENDERS resulta em conjuntos de testes mais fortes do que aqueles produzidos por ferramentas automatizadas.

\section{Relação entre Processos de Produção e Técnicas de Crowdsourcing}

A relação entre as etapas de desenvolvimento e os meios de crowdsourcing foram realizadas partindo de formas possíveis de se aplicar o crowdsourcing dentro de cada uma das etapas de desenvolvimento, levando em consideração os artefatos que deveriam ser produzidos durante a etapa específica, o grau de publicização do projeto e as práticas já existentes na indústria de incluir o usuário no ciclo de desenvolvimento.

Um processo de desenvolvimento de um jogo digital passa por diversas etapas [IGN 2006]. Estruturados de modo diferente por diversos autores, empresas e equipes de desenvolvimento, o objetivo dos processos se mantém como etapas que devem ser executadas em ordem para que a criação do produto final seja feita do modo mais eficiente. Comumente esse processo envolve as seguintes etapas:

- Conceito: momento no qual os desenvolvedores estão focados no que é a proposta para o produto, quais seus principais pontos em relação aos concorrentes e sua ideia central.

- Pré-Produção: é o momento no qual a proposta do jogo é validada e os primeiros elementos são criados para se alinharem ideias tanto na implementação quanto nos aspectos artísticos e de experienciais do jogo.

- Produção: é quando ocorre a implementação propriamente dita, com um projeto já estabelecido nas etapas anteriores e objetivos bem definidos para serem cumpridos por todos os profissionais envolvidos. 
- Pós-Produção: etapa na qual ocorrem os testes de versões públicas do jogo, adequação aos requerimentos de plataformas específicas, ajustes eventuais de problemas e, por fim, o lançamento.

Desde 2006, quando o termo crowdsourcing foi cunhado por Jeff Howe, seu uso vem se tornando cada vez mais abrangente para definir meios de compartilhamento de trabalho e esforço coletivo para atingir um objetivo [Howe 2006]. Tal tática é utilizada em meios mais tradicionais como o de comércio e serviços, assim como em aplicativos. Dentro dessas diversas etapas do processo de desenvolvimento, é possível haver a intrusão de participantes externos ao desenvolvimento, oferecendo colaborações tanto na validação de conceitos apresentados pelos desenvolvedores quanto de forma mais prática, desenvolvendo em conjunto com a equipe o produto final.

Dentro da indústria de jogos, cada uma das etapas do processo de desenvolvimento pode assimilar diversas formas de crowdsourcing. Para os desenvolvedores, essas táticas podem se tornar interessantes em detrimento de formas mais tradicionais de conclusão de projetos. Do mesmo modo que a maior parte das empresas que trabalham desenvolvendo produtos para o usuário final, a aplicação do crowdsourcing pode refletir os mesmos benefícios levantados, como a redução dos custos de desenvolvimento, resolução de problemas aliado ao feedback constante dos usuários, além de ser uma forma de criar uma relação mais próxima entre o usuário e o produto.

Há diversos casos de aplicação de meios de crowdsourcing em jogos, os quais foram inseridos no conceito, trabalhados durante o desenvolvimento ou surgiram após o lançamento. Esses casos vão do uso da base de usuários para a definição de temas e conceitos abordados no jogo, algo próprio de fases iniciais do desenvolvimento, passando por feedbacks iterativos durante o processo de desenvolvimento mirando a qualidade do produto final até o estabelecimento de meios de modificação e criação baseados no jogo original em um momento posterior ao lançamento do jogo.

Dentro da etapa de Conceito, momento no qual são definidas as principais características do projeto, é muito comum a utilização de meios de questionário e pesquisas de opinião para validar a recepção da ideia diante do público. Da mesma forma que em outras áreas, onde ter certeza que o produto em desenvolvimento possui apelo e satisfaz as necessidades de seu cliente ou público, como o Design Gráfico e o Desenvolvimento de Sistemas, na indústria de jogos tal validação pode decidir todo o futuro do projeto.

Um exemplo de aplicação crowdsourcing durante a etapa de Pré-Produção de um jogo está na forma de aproximar os usuários de seu processo de desenvolvimento, criando projetos que possam ser facilmente customizáveis conforme o feedback de jogadores por meio de canais de comunicação e mídias sociais.

Durante a Produção de um jogo, como o foco está totalmente na implementação e funcionalidades, o que se pode focar para crowdsourcing está na criação de conteúdo e no feedback dos usuários sobre o que foi criado. Há práticas comuns na indústria, como a disponibilização de elementos conceituais, teasers e testes de conceito produzidos durante o desenvolvimento, especialmente em casos que o financiamento do jogo partiu do público, com o uso de canais de crowdfunding, por exemplo, o que são casos em que o desenvolvedor e o consumidor possuem uma relação mais próxima de financiador e prestador de serviço. 
A Pós-Produção é a última fase do desenvolvimento de um jogo, envolvendo etapas de teste, validação e lançamento de um projeto, e período pós-lançamento, no qual as empresas costumam manter um suporte ativo ao produto recém-lançado em busca de corrigir problemas e adicionar novos conteúdos. Durante a etapa que o jogo ainda está para ser lançado, normalmente os trabalhos são corretivos e a contribuição que a comunidade oferece é no suporte e validação do jogo por meio de testes.

\section{Conclusão}

Esta pesquisa tem como objetivo realizar uma avaliação do impacto do uso de estratégias de crowdsourcing no desenvolvimento e manutenção do engajamento em jogos digitais, considerando etapas gerais do desenvolvimento de jogos, que são: Conceito, PréProdução, Produção e Pós-Produção. O estágio da pesquisa ainda é inicial, e tem como próxima etapa uma análise de casos reais de jogos que utilizaram crowdsourcing de alguma forma em seu desenvolvimento.

\section{Referências}

Carter, S., Smith, M., Bali, S., Sotiriadis, S., Bessis, N., e Hill, R. (2012). The use of crowdsourcing to aid quest design in games. In 2012 Third International Conference on Emerging Intelligent Data and Web Technologies, pages 302-305.

Howe, J. (2006). The rise of crowdsourcing - wired. https://www.wired.com/2006/06/crowds/. Online; acessado em março-2018.

Howe, J. (2008). Crowdsourcing: How the Power of the Crowd is Driving the Future of Business. Great Britain: Business Books.

$\mathrm{Hu}, \mathrm{Z}$. e Wu, W. (2014). A game theoretic model of software crowdsourcing. In 2014 IEEE 8th International Symposium on Service Oriented System Engineering.

IGN (2006). The game production pipeline: Concept to completion. http://www.ign.com/articles/2006/03/16/the-game-production-pipeline-conceptto-completion. Online; acessado em março-2018.

Munezero, M., Kakkonen, T., Sedano, C. I., Sutinen, E., e Montero, C. S. (2013). Emotionexpert: Facebook game for crowdsourcing annotations for emotion detection. In 2013 IEEE International Games Innovation Conference (IGIC), pages 179-186.

Prandi, C., Salomoni, P., Roccetti, M., Nisi, V., e Nunes, N. J. (2016). Walking with geo-zombie: A pervasive game to engage people in urban crowdsourcing. In 2016 International Conference on Computing, Networking and Communications (ICNC).

Rojas, J. M., White, T. D., Clegg, B. S., e Fraser, G. (2017). Code defenders: Crowdsourcing effective tests and subtle mutants with a mutation testing game. In Proceedings of the 39th International Conference on Software Engineering, ICSE'17, pages 677-688, Piscataway, NJ, USA. IEEE Press.

Shaker, N., Yannakakis, G. N., e Togelius, J. (2013). Crowdsourcing the aesthetics of platform games. IEEE Transactions on Computational Intelligence and AI in Games, 5(3):276-290.

Wang, A., Hoang, C. D. V., e Kan, M.-Y. (2013). Perspectives on crowdsourcing annotations for natural language processing. Language Resources and Evaluation, 47(1):9-31. 